\title{
Teoría y práctica en Musonio Rufo: Un análisis crítico de las Disertaciones 5 y 6
}

\author{
Theory and practice in Musonio Rufo: \\ A critical analysis of Lectures 5 and 6
}

RODRIGO SEBASTIÁN BRAICOVICH

Consejo Nacional de Investigaciones Científicas y Técnicas (CONICET) (Argentina)

\section{RESUMEN}

Los objetivos específicos son los siguientes: (i) reconstruir en forma sistemática la relación entre

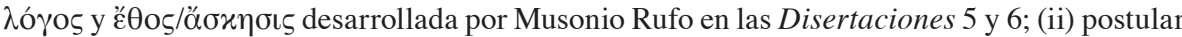
las reflexiones de Aristóteles sobre el problema de la habituación como un marco conceptual relevante para encuadrar el análisis de ambas disertaciones; (iii) analizar las posibles tensiones

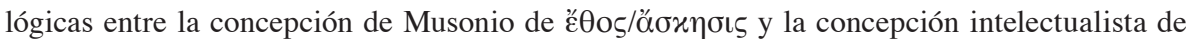
la acción humana defendida por la ortodoxia estoica. Sugeriré asimismo que el recurso a las Dissertationes de Epicteto puede ofrecer una respuesta tentativa a interrogantes no resueltos en las Disertaciones 5 y 6.

PALABRAS CLAVE

EJERCICIOS ESPIRITUALES, ESTOICISMO, INTELECTUALISMO, PSICOLOGÍA DE LA ACCIÓN

\section{ABSTRACT}

The specific goals are the following: (i) to put together in a systematic manner the relationship

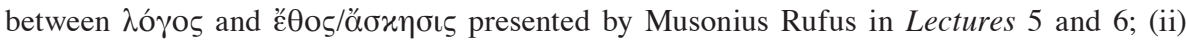


to propose Aristotle's reflections on the problem of habituation as a relevant framework to make sense of both lectures; (iii) to analyze the possible logical conflicts between Musonius'

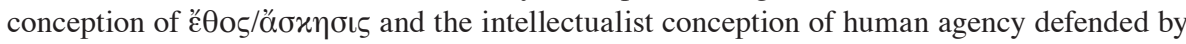
Stoic orthodoxy. I will further suggest that Epictetus' Discourses may offer a tentative answer to questions that are left unanswered in Lectures 5 and 6.

\section{KEYWORDS}

SPIRITUAL EXERCISES, STOICISM, INTELLECTUALISM, PSYCHOLOGY OF ACTION

\section{INTRODUCCIÓN}

Si BIEN LAS DISERTACIONES QUe SE HAN PRESERVADo de Musonio Rufo a partir del registro de Lucio, uno de sus alumnos, ${ }^{1}$ constituyen el capítulo más inexplorado del período romano de la escuela estoica, uno de los dos elementos por los que dichas disertaciones suelen ser citadas está representado por la defensa que Musonio realiza de la necesidad de concebir la filosofía en términos eminentemente prácticos. ${ }^{2}$ El tipo de abordaje que se realiza, a este respecto, cubre desde la alusión ocasional a la primacía que el autor habría otorgado a la práctica por sobre la teoría, hasta la inclusión explícita de Musonio en una corriente de pensadores que intentan distanciarse de la concepción esencialmente contemplativa que el aristotelismo propone de la filosofía para construir una alternativa primordialmente práctica, centrada en ciertos «ejercicios espirituales» que pasarían a ocupar el rol central en el diseño de la praxis pedagógica de la filosofía. ${ }^{3}$

El objetivo general de estas páginas consistirá en analizar las Disertaciones 5 y 6 de Musonio [D5 y D6, de ahora en más], ${ }^{4}$ concentrándome en los siguientes

1 G. Roskam 2005:99-100 ofrece un abordaje sintético de la problemática de la conservación de las disertaciones de Musonio.

2 El otro elemento por el que se suele recurrir a Musonio está representado por su posición respecto de los roles y derechos de la mujer en la sociedad.

3 Tal es el caso de la lectura propuesta por John Sellars (vid. J. Sellars 2003 y 2007), inspirada fundamentalmente en las interpretaciones previas de Pierre Hadot y Michel Foucault.

4 Utilizaré, tanto para las citas en griego como para las referencias, la edición de Cora Lutz (C. Lutz 1947). Las traducciones al español son propias. 
objetivos específicos: $i$ ) reconstruir el concepto de habituación/ejercitación (‘ُقo /

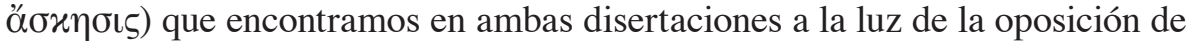
ambas nociones al $\lambda$ ó $\gamma \mathrm{os}$, entendido como comprensión teórica de principios filosóficos; ii) traer a la luz el posible trasfondo aristotélico (y no únicamente estoico) de la distinción entre č $\theta$ o 5 y $\lambda$ ó

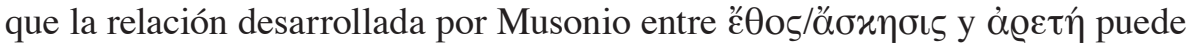
generar respecto de la concepción intelectualista de la acción defendida por el estoicismo antiguo. Como estrategia hermenéutica, por último, sugeriré que ciertas lagunas dejadas por argumentación de Musonio en D5-6 pueden ser

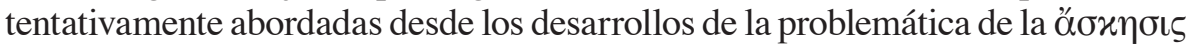
que encontramos en las Dissertationes de Epicteto.

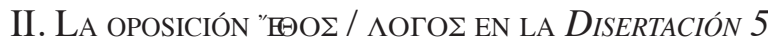

El objetivo de la Disertación 5 conservada por Lucio/Estobeo se encuentra establecido en forma sencilla al inicio de la misma:

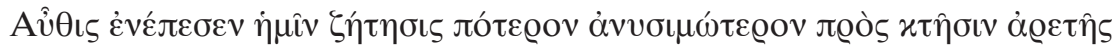

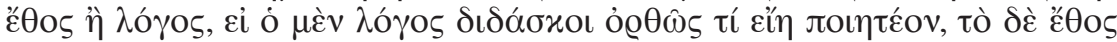

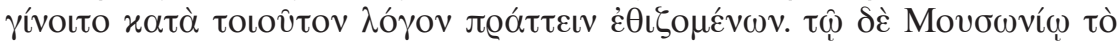

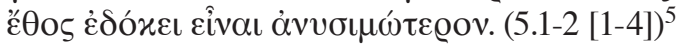

En otra ocasión cierto surgió entre nosotros la pregunta acerca de qué era más efectivo para alcanzar la virtud, el hábito o la teoría, dado que la teoría enseña qué es correcto hacer, mientras que el hábito surge en aquellos que están acostumbrados a actuar de acuerdo con dicha teoría. Musonio opinaba que el hábito era más efectivo.

Los pasajes siguientes (5.3-10) representan un contrapunto entre dos tipos de individuos mediante el cual Musonio pretende ofrecer razones en favor de la afirmación volcada en 5.2: la primera alternativa consiste en un médico/navegante/músico que es capaz de hablar versadamente (ixavov̂ $\lambda{ }^{\prime} \gamma \varepsilon ı v$ ) sobre los principios teóricos de su arte (medicina/ navegación/música), pero que carece de experiencia en llevar dichos principios a la práctica; la segunda alternativa consiste en un médico/ navegante/músico que no posee la capacidad de hablar versadamente ${ }^{6}$

5 En lo sucesivo, las referencias a las disertaciones de Musonio siguen el siguiente formato: (número de disertación.sección de Lutz [líneas en la edición de Lutz])

6 En el caso del navegante, Musonio presenta la alternativa de un individuo que es capaz

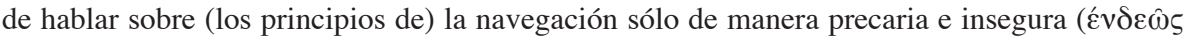

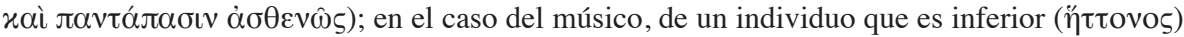
en su conocimiento de la teoría de la música que su contraparte. 
sobre dichos principios teóricos pero que posee experiencia en llevarlos a la práctica. Luego de presentar ambas alternativas, Musonio interroga a su interlocutor acerca de cuál de las dos elegiría en cada caso, ante lo cual éste responde sistemáticamente en favor de la segunda opción. A partir de lo establecido hasta aquí, y luego de extender la analogía al caso de la

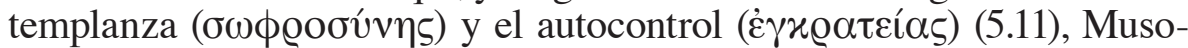
nio deduce una serie de conclusiones pretendidamente equivalentes $\mathrm{o}$, al menos, mutuamente implicadas:

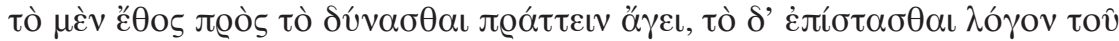

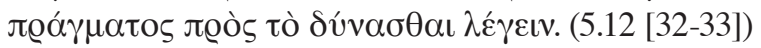

El hábito nos conduce a la capacidad para actuar, pero conocer la razón detrás de los actos nos permite hablar sobre esos actos.

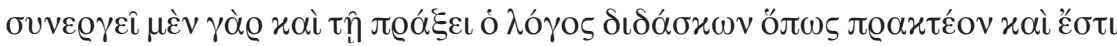

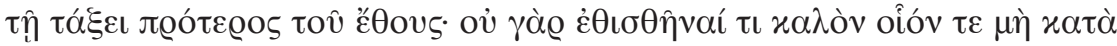

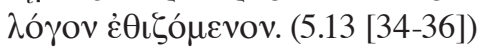

La teoría que nos enseña cómo actuar opera junto con la acción y la precede, dado que no es posible acostumbrarse a hacer algo bueno a menos que nos acostumbremos a hacerlo de acuerdo con la teoría.

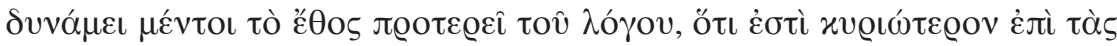

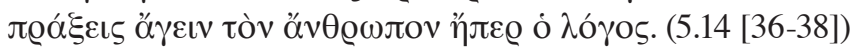

Sin embargo, lo cierto es que el hábito es superior a la teoría, en la medida en que es más efectiva para conducir a los hombres a actuar que la teoría.

Al considerar la totalidad de la disertación, se abren dos posibles líneas de interpretación acerca de cuál es su objetivo último: si nos concentramos fundamentalmente en las analogías provistas por Musonio, el motivo central de esta disertación parece ser la crítica (frecuente asimismo en Epicteto) a aquellos que pronuncian discursos públicamente y hacen gala de sus profundos conocimientos de la teoría de una escuela y, sin embargo, no actúan siguiendo esos principios, una idea que aparecía en forma explícita ya en D3: «ő блє@

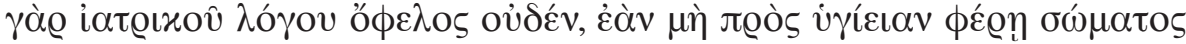

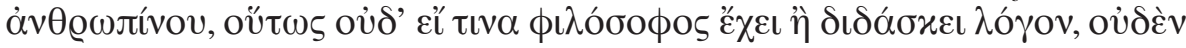




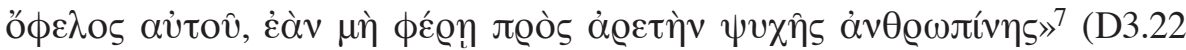
[63-65]). Si nos atenemos, por el contrario, a la discusión planteada en D5.1-2 y 5.12-14, parece evidente que ese no es el motor real de la disertación (no al menos el motor central), y que la contraposición entre la mera capacidad de hablar $(\lambda \varepsilon \dot{\varepsilon} \varepsilon v v)$ sobre una teoría y la capacidad de poner en obras dicha teoría es, a fin de cuentas, secundaria respecto de la distinción entre conocer la teoría (independientemente de que podamos pronunciar discursos acerca de la misma) y actuar de acuerdo con ella.

Ambos objetivos no son, sin embargo, inconciliables, y podemos suponer que D5 está atravesada en su totalidad por dos ideas complementarias: i) el mero conocimiento de una teoría es suficiente para hablar versadamente sobre ella, pero no para actuar de acuerdo con la misma; ii) para actuar de acuerdo con una teoría el conocimiento de la misma debe ser complementado con el hábito, la práctica reiterada de cierto tipo de acciones. Esta interpretación ha sido efectivamente adoptada por buena parte de los comentaristas de Musonio, y ha sido señalada en ciertas ocasiones como el momento inicial en una nueva forma de concebir la filosofía: una concepción que, distanciándose radicalmente de la matriz contemplativa de la filosofía aristotélica, concibe la dimensión práctica de la tarea filosófica como igual, o incluso como más importante, que la incorporación de la teoría. ${ }^{8}$ A pesar de ello, la aparente transparencia hermenéutica de D5 depende fundamentalmente de traducciones ciertamente cuestionables, y un análisis de las fuentes griegas revela que la superficie clara de D5 esconde en realidad dificultades lógicas sustantivas. Que esto es así se hace evidente si atendemos al esquema argumental de la disertación:

5.1: $\quad$ Planteo de la pregunta: ¿qué es más efectivo para adquirir la virtud, la teoría o el hábito? [p1]

5.2: $\quad$ Respuesta a p1: el hábito es más efectivo que la teoría para adquirir la virtud [r1];

5.3-10: Razones en favor de r1;

5.11: A partir de la evidencia de 5.3-10, Musonio concluye que es mejor ser temperado que poder hablar sobre la templanza [r1'];

5.12: $\quad$ A partir de lo dicho en 5.1-11, Musonio concluye que conocer la teoría de algo nos permite hablar sobre eso, pero es el hábito el que nos permite actuar de acuerdo a la teoría [r2], y que

7 «Así como la teoría médica no tiene ninguna utilidad si no conduce a la salud del cuerpo del hombre, que un filósofo enseñe su teoría no tiene ninguna utilidad si no conduce a la virtud del alma del hombre».

$8 C f$. paradigmáticamente J. Sellars 2003. Cf. Asimismo G. Reydams-Schils 2005:156; G. Roskam 2005:100; M. Morford 2002:195, quienes aceptan (quizás tomando a la letra la traducción de Lutz), la idea de que la práctica es más importante que la teoría. 
5.13: a pesar de que la teoría es anterior al hábito en tanto no es posible actuar correctamente si no conocemos la teoría [r3], 5.14: el hábito es anterior a la teoría en tanto es más efectivo para conducir a los hombres a la acción [r4].

A pesar de que las dificultades aquí presentes son múltiples, me interesa detenerme únicamente sobre tres elementos: en primer lugar, y como se desprende de los ejemplos proporcionados por Musonio en D5.3-10, la disyuntiva allí planteada es falsa, en la medida en que, como se indica en D5.13 (y en otros pasajes que veremos más adelante), el hábito no es posible sin la teoría. De lo que se trata, en otras palabras, no es de elegir entre la teoría y el hábito, sino de elegir entre solamente la teoría o, por el contrario, la teoría y el hábito (de actuar de acuerdo con la misma ${ }^{9}$ ). Como se deduce de D8.710 y como se verá en D6, el hábito no puede darse sin la teoría, y si esto es así, la pregunta de Musonio acerca de si el hábito es más efectivo que la teoría (p1) carece por completo de sentido, en tanto el hábito es hábito de actuar de acuerdo con una teoría, es decir: la puesta en acción de ciertos principios teóricos. La pregunta que Musonio debería formular -y que de hecho va a formular explícitamente en D6- no es si la teoría es más efectiva que el hábito, sino si aquella es suficiente, sin el hábito, para alcanzar la virtud. Una segunda dificultad se refiere exclusivamente a D5.14, y consiste en el hecho de que $r 4$ no es una respuesta a pl, y la diferencia entre $\mathrm{rl}$ y $\mathrm{r} 4$ es sustantiva: afirmar que el hábito es más efectivo para conducir a los hombres a la acción (r4) no es lo mismo que afirmar que es más efectivo para alcanzar la virtud (r1). Aun si concediéramos -caritativamente- a Musonio que a través de D5.3-10 ha demostrado r4, eso no significa que haya demostrado r1, como pretende haber hecho. En rigor, a diferencia de r1 (que, como veremos, adquiere una dimensión plena cuando la interpretamos

9 Se pone en cuestión, con todo esto, la adecuación de las analogías proporcionadas por Musonio, en la medida en que, contra lo afirmado en D5.13, al menos en el caso de la música y la navegación es efectivamente posible ser capaz y experimentado en ambas artes sin ser conocedor de la teoría. No es esta posibilidad la que tiene en mente Musonio: tanto en el caso de la música como de la navegación, el contrapunto no se produce entre el conocedor de la teoría y que puede hablar versadamente sobre ella y el que no puede hablar en absoluto acerca de la teoría, sino entre aquél y el que es inferior en la teoría musical o el que puede hablar de manera precaria sobre la teoría de la navegación; en ninguno de los dos casos, en suma, se trata de posesión versus carencia de cierto conocimiento, sino más bien grados de conocimiento.

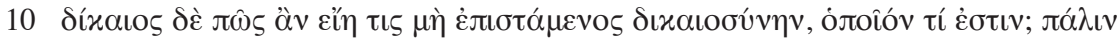

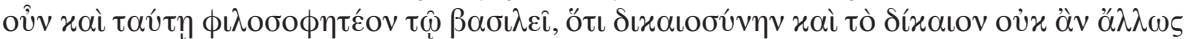

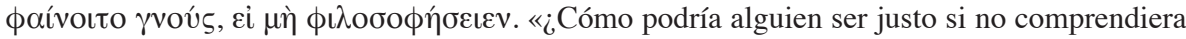
qué es la justicia? Es por ello que el rey debe estudiar filosofía, dado que, si no lo hiciera, no estaría claro si sabe qué es la justicia y lo justo» (D8.7 [22-25]). 
en el contexto conceptual apropiado) r4, sin especificaciones adicionales, carece por completo de sentido. Ninguna de estas dos dificultades, sin embargo, resulta decisiva, en la medida en que ambas pueden ser explicadas apelando al contexto esencialmente pedagógico en el que se inscriben las disertaciones recogidas por Lucio: ${ }^{11}$ desde esa perspectiva, tanto la consideración de $\varepsilon^{\prime} \theta$ o como un aspecto más importante que $\lambda$ ó $\gamma$ o $\varsigma$ como el pasaje de r1 a r4 no pasan de ser meras estrategias retóricas, cuya intención consiste en transmitir en forma provocativa la idea de que la teoría, aun cuando sea necesaria, no es suficiente para alcanzar la virtud. ${ }^{12}$

Existe, no obstante, un tercer problema: aun si interpretamos $\mathrm{r} 4$ como una variante retórica de $\mathrm{r} 1$, ambos exigen una explicación, en la medida en que plantean una dificultad que no puede ser desarticulada -como en los otros casos- recurriendo al contexto pedagógico y nos exige indagar más allá. La dificultad en cuestión se reduce a la siguiente pregunta: ¿cómo es posible que estar habituado a hacer $x$ sea condición de posibilidad de hacer $x$ ? Vg.: ¿en qué sentido estar habituado a actuar templadamente puede ser condición de posibilidad de actuar templadamente ( $^{13}$ Para comprender lo que se halla en juego aquí, no obstante, debemos antes abordar la discusión complementaria que Musonio desarrolla en D6.

$11 C f$., a este respecto, G. Roskam 2005:100-101; Ch. Pomeroy Parker 1896:126-127.

12 Si bien estas dos dificultades pueden parecer triviales en vistas de la solución propuesta, la confusión que ellas suscitan es el punto de partida de la lectura ofrecida por Sellars respecto de la esencialidad de la práctica en Musonio (centralidad que Sellars proyecta a Epicteto y Marco Aurelio). D8 ofrece evidencia adicional respecto de esta necesidad de una interpretación enmarcada en las intenciones pedagógicas que guían cada disertación, dado que allí encontramos

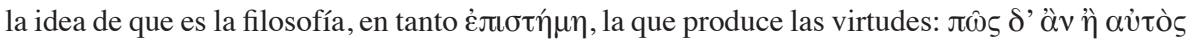

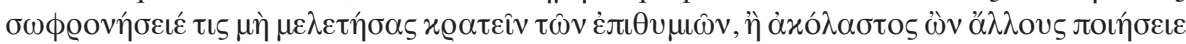

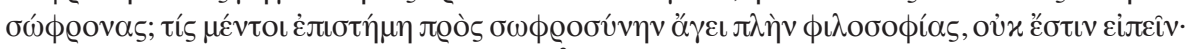

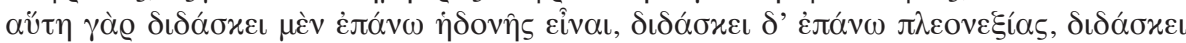

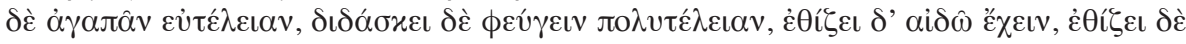

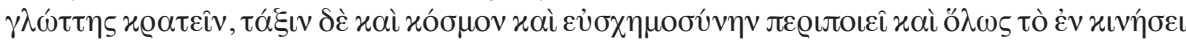

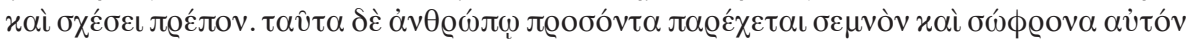
(D8.15-17 [42-50]). Lo decisivo aquí es que, a diferencia de D5-6, donde uno de los objetivos centrales consiste en convencer al interlocutor acerca de la necesidad de no limitarse al estudio de la filosofía, el objetivo de D8 consiste en convencer a un rey respecto de la necesidad de (al menos) iniciarse en el estudio de la misma si desea volverse un gobernante justo.

13 Optar por traducir ع̈Өos por «práctica», como hacen Lutz y King, no representa ninguna solución, en tanto afirmar que solo podremos hacer $x$ si antes hemos practicado $x$ carece igualmente de sentido. 


\section{LA OPOSICIÓN ’A $\mathrm{KKH} \Sigma \mathrm{I} \Sigma / \Lambda$ / $\mathrm{O} \mathrm{O} \Sigma$ EN LA DISERTACIÓN 6}

El inicio de la Disertación 6 da cuenta de la continuidad temática y argumental respecto de D5:

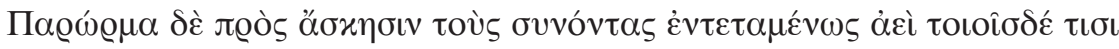

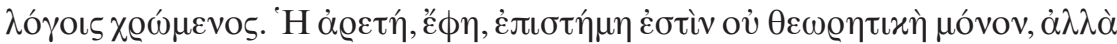

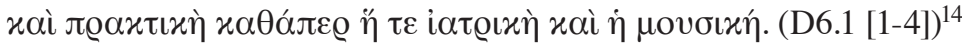

Siempre incitaba con vehemencia a los que lo rodeaban a que se ejercitasen usando discursos como éste: la virtud, decía, no es solamente conocimiento teórico sino también práctica, tal como lo son la medicina y la música.

A pesar de que los cinco parágrafos siguientes evidencian un giro terminológico importante, en la medida en que desplazan el eje de la discusión desde

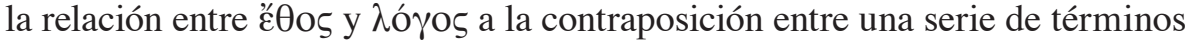
vinculados a la comprensión o el aprendizaje de principios teóricos, por un lado, y la ejercitación ${ }^{15}$ de dichos principios, por otro, el uso reiterado de $\dot{\varepsilon} \theta i ́ \zeta \omega$ como equivalente de esta segunda serie de términos justifica que asumamos

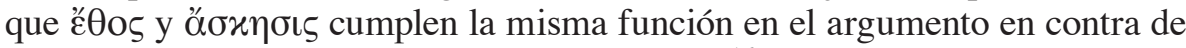
la suficiencia del $\lambda$ ó $\gamma$ os para alcanzar la virtud. ${ }^{16}$ Como se puede ver en el siguiente esquema, la idea fundamental que atraviesa D6 sigue siendo la misma que encontrábamos en D5.1-2 y 11-14:

6.2: Para ser bueno es necesario no sólo aprender en profundidad los principios $(\mu \alpha \theta \eta \mu \alpha \tau \alpha)$ que conducen a la virtud sino

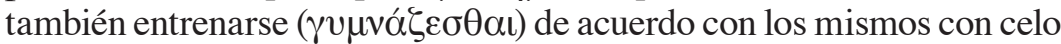
y rigor.

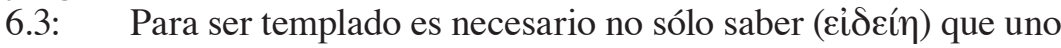
no debe ser derrotado por los placeres sino también estar entrenado en oponerse a los mismos.

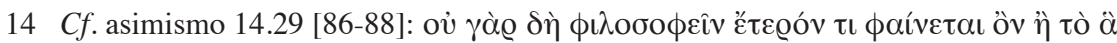

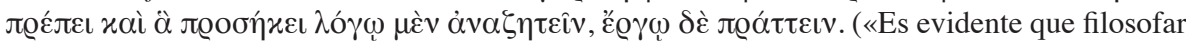
no es otra cosa que investigar mediante la razón qué es apropiado y correcto y transformarlo en obras»).

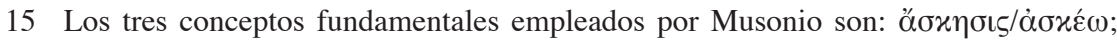

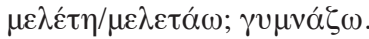

16 Esto no hace menos cuestionable, no obstante, la decisión de Cora Lutz y Cynthia King de traducir «ै 0 os por «practice» en D5 (estableciendo una absoluta homogeneidad conceptual entre ambas disertaciones), en la medida en que tal decisión contribuye a ocultar uno de los problemas centrales que señalé anteriormente, a saber: ¿cómo es posible que Musonio suponga que estar habituado a hacer $X$ es condición de posibilidad de hacer $X$ ? 
6.4: $\quad$ Para ser justo es necesario no solo aprender ( $\mu \varepsilon \mu \alpha \theta \eta \chi \omega े \varsigma)$ que

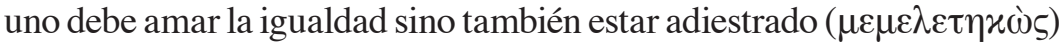
en evitar los excesos.

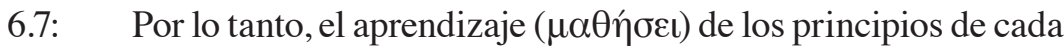

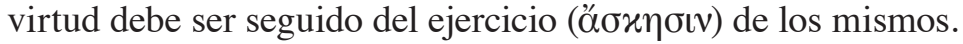

Ahora bien: ¿Cuál es la diferencia entre hacer $x$ y entrenarse para hacer $x$ ? ¿Qué características debe poseer un acto que realizo para que sea considerado como un acto en sí mismo y no un ejercicio para el mismo? En otras palabras: ¿por qué el entrenamiento no es ya la acción?

La referencia a Aristóteles, llegados a este punto, se vuelve ineludible:

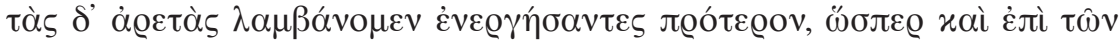

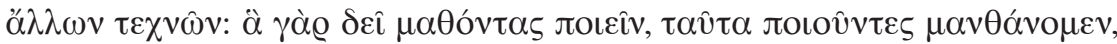

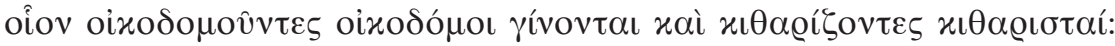

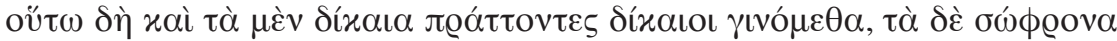

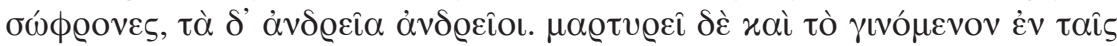

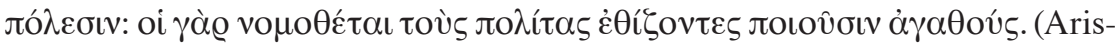
tóteles, EN 1103a32-1103b3)

Adquirimos las virtudes después de ejercerlas primero, como es el caso también en las demás artes, pues las aprendemos haciendo lo mismo que se debe hacer después de haberlas aprendido; por ejemplo, se llega a ser constructor de casas construyendo casas, y citarista, tocando la cítara. De ese modo, pues, también llegamos a ser justos realizando actos justos, moderados realizando actos de moderación, y valientes realizando actos de valentía. [Lo] atestigua también lo que sucede en las ciudades, esto es, que los legisladores hacen buenos a los ciudadanos acostumbrándolos [a serlo]. ${ }^{17}$

Las dificultades presentes en este célebre pasaje de $E N$ son parcialmente análogas a las señaladas respecto de D5: en primer lugar, la elección de ejemplos por parte de Aristóteles adolece del mismo problema que señalábamos anteriormente respecto del pasaje de Musonio de r1 a r4, a saber, que la idea de que

(a) hacer $x$ (vg.: construir casas) en repetidas ocasiones hace posible hacer $x$;

no es lo mismo que la idea de que:

(b) hacer $x$ (vg.: construir casas) en repetidas ocasiones hace posible hacer $x$ virtuosamente.

17 Cito de acuerdo con I. Bywater 1962; la traducción es de E. Sinnott (Aristóteles 2007). 
Sin especificaciones adicionales, (a) carece por completo de sentido.

En segundo lugar, la idea de que adquirimos una virtud después de ejercerla representa, prima facie, un contrasentido tan evidente como el que indicábamos en el caso de D5.14. Aristóteles, sin embargo, y a diferencia de Musonio, se anticipa inmediatamente a dicha objeción:

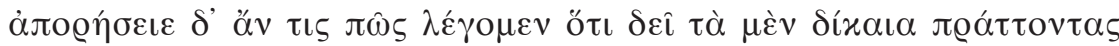

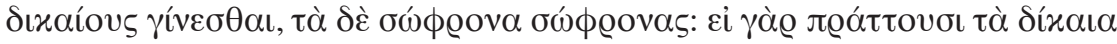

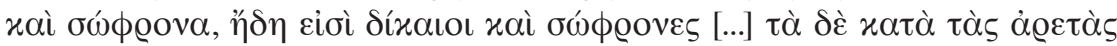

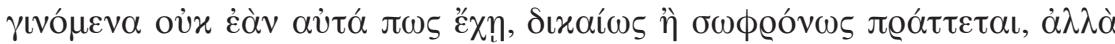

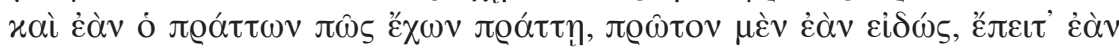

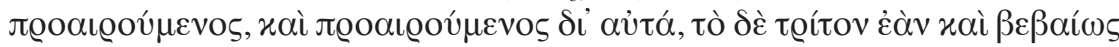

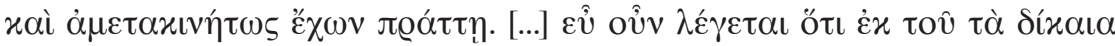

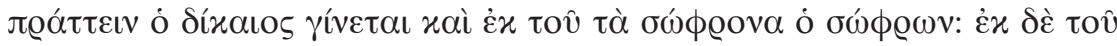

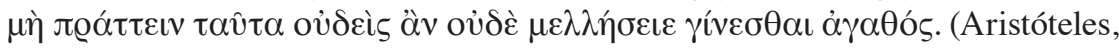
EN 1105a17-1105b13)

Se podría plantear la dificultad de cómo decimos que se debe llegar a ser justo realizando actos justos, y moderados realizando actos moderados, pues si se realizan actos justos y moderados, ya se es justo y moderado. [...] Las acciones originadas de acuerdo con las virtudes no se realizan de manera justa o moderada solo si son de un cierto modo, sino también si el que actúa se halla [dispuesto] de un cierto modo cuando actúa: primero, si [actúa] a sabiendas; después, [si actúa] eligiendo, y eligiendo [el acto] de por sí; y, tercero, si actúa de manera firme e inconmovible. [...] Es, pues, acertado decir que el justo llega a ser tal por realizar actos justos, y el moderado, por [realizar] actos moderados; y si no realiza esos actos, nadie tiene siquiera la posibilidad de llegar a ser bueno. Con todo, el común de los hombres no practica esas acciones, sino que, refugiándose en la teoría, creen que filosofan, y que así llegarán a ser virtuosos. ${ }^{18}$

18 ¿Leyó Musonio EN? Varios elementos en los dos pasajes citados parecerían sugerir una lectura directa de Aristóteles por parte de Musonio: (i) la analogía con el citaredo, (ii) la

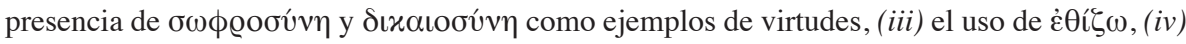
la idea de que nos volvemos virtuosos mediante la práctica de las virtudes, y $(v)$ la crítica a los que creen que el conocimiento de la teoría es suficiente para alcanzar la virtud. Si bien la coincidencia entre Aristóteles y Musonio respecto de cada uno de estos elementos no indica nada si los consideramos por separado, la coexistencia de todos estos puntos parecería representar evidencia suficiente respecto de una influencia directa. Todo ello, sin embargo, es meramente circunstancial: la cítara como objeto de referencia en las analogías entre la filosofía y otras artes no contribuye en absoluto un patrimonio exclusivo de Aristóteles, sino que es frecuente en la filosofía antigua; la convivencia de (iii) y (iv), por su parte, bien puede proceder de cualquier tratado peripatético y no requiere suponer necesariamente la lectura directa de EN 1103a32- 
Los elementos fundamentales en este pasaje son dos: en primer lugar, la distinción entre (a) la realización de un acto justo (moderado, valiente, etc.) y (b) la realización de ese mismo acto tal como lo realizaría un hombre virtuoso (lo cual incluye la disposición del agente); en segundo lugar, la idea de que la realización repetida de $(a)$ puede conducir a $(b)$. Si bien la primera idea es esencialmente incontestable (siempre y cuando supongamos la existencia de algo así como la virtud), es la segunda idea la que ha generado importantes polémicas entre los comentaristas de Aristóteles a lo largo de la historia de Occidente, fundamentalmente en lo que respecta a las características específicas del proceso de habituación. La pregunta central ha sido, una y otra vez, la siguiente: ¿cómo es posible que la mera repetición de ciertos actos (o el mero hábito de realizarlos) sea causante de un eventual cambio en la disposición psíquica del agente? ${ }^{19} \mathrm{Si}$ bien existe un consenso relativamente extendido entre los comentaristas en cuanto a que el proceso de habituación al que hace referencia Aristóteles no constituye un proceso de repetición puramente mecánico, a partir del cual el cambio de disposición en el alma del agente sobrevendría de un modo cuasi mágico, ${ }^{20}$ no existe un consenso claro acerca de lo que sí se encuentra implicado en dicho proceso. ${ }^{21} \mathrm{El}$ análisis de este problema excede, sin embargo, los objetivos del presente artículo, y no representa un obstáculo para la argumentación general, en la medida en que la referencia a Aristóteles solo tenía por objetivo presentar un contexto posible en el cual enmarcar la discusión que articula D5 y D6.

Se podría argumentar, no obstante, que la referencia a Aristóteles no era en absoluto necesaria, en la medida en que los desarrollos teóricos del estoicismo antiguo ofrecen una alternativa conceptual (parcialmente) análoga a la distinción aristotélica entre el acto en sí mismo y el acto tal como lo realizaría el hombre virtuoso, a saber, la distinción entre los conceptos de $x \alpha \theta \eta \dot{x o v} \tau \alpha$ y $\varkappa \alpha \tau o ́ \varrho \theta \omega \mu \alpha .{ }^{22}$ Podemos, por ejemplo, componer a partir de los testimonios

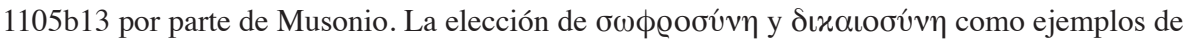
virtud, por último, tampoco requiere de la lectura de tal pasaje, en la medida en que las mismas, además de ser dos de las cuatros virtudes cardinales estoicas, aparecen en forma recurrente a lo largo de las disertaciones de Musonio en conjunción con otras virtudes.

19 Un tratamiento reciente de esta problemática específica se encuentra en N. Bowditch 2008; $c f$. asimismo S. Broadie 1991:103-110.

20 Cf., entre otros, P. Gottlieb 2009:186; R. Sorabji 1973:124-127; R. Hursthouse 1998:210-212; N. Bowditch 2008:315.

21 Para una interpretación esencialmente cognitivista del problema, vid. R. Sorabji 1973.

$22 C f$., fundamentalmente, Stob. 2.85,13-86,4 [LS 59B; SVF 3.494]; Cic. Fin. 3.58-9 [LS 59F] y 3.24-5 [LS 64H; SVF 3.11]; Philo Cher. 14-15 [LS 59H; SVF 3.513]; Stob. 2.93,14-18 [LS 59K; SVF 3.500]. Para una discusión general de este problema, $c f$. B. Inwood 1985: 205-215. 
legados por Cicerón y Estobeo una imagen mínima de lo que los estoicos antiguos pudieron haber entendido por tal distinción: si atendemos a Cicerón,

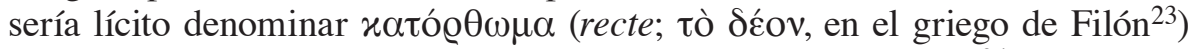
a una acción que ha sido realizada 'de la forma correcta' (iuste ${ }^{24}$ ) y contiene

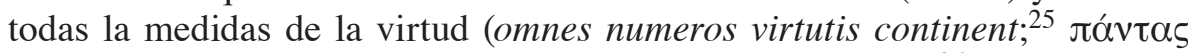

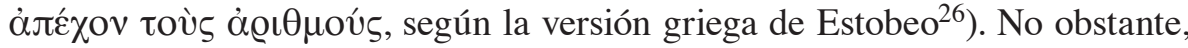

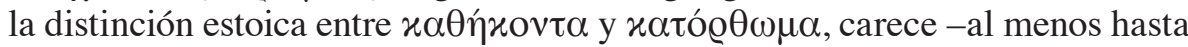
donde sabemos- de un elemento que resulta central para D5 y D6, a saber, la idea de la habituación o ejercitación, y es precisamente ese elemento el que me interesa resaltar.

\section{LA IDEA DE HABITUACIÓN/EJERCICIO EN EL HORIZONTE INTELECTUALISTA ESTOICO}

Fundamentalmente a partir de los estudios de Pierre Hadot en las décadas de 1960 y 1970, un aspecto que ha sido reiteradamente señalado por los comentaristas como uno de los aportes decisivos del período romano de la escuela estoica (fundamentalmente tomando en consideración a Séneca, Musonio, Epicteto y Marco Aurelio) consiste en la dimensión esencialmente práctica del abordaje que tales autores ofrecen de la filosofía. Trascendiendo el mero 'giro hacia la ética' que se verifica en dicho período, las obras que se han conservado de los estoicos romanos representan un repertorio compuesto por una serie de «ejercicios espirituales» destinados a poner al proficiente en camino hacia la virtud: desde el aprendizaje memorístico hasta la repetición incesante de ciertos principios teóricos, pasando por ejercicios específicos como abstenerse de beber agua o de ingerir comida, el estoicismo romano ofrecería -de acuerdo con dicha línea de lectura- una visión más realista y cotidiana de la labor filosófica que la alternativa ofrecida por los desarrollos lógicos y físicos de Crisipo o, yendo más atrás, por la concepción esencialmente teorética de la filosofía desarrollada por Aristóteles. ${ }^{27}$

Ahora bien, el énfasis -muchas veces descontextualizado ${ }^{28}$ - sobre este tipo de ejercicios ha impedido percibir claramente hasta qué punto ese tipo de estrategias puede constituir una amenaza directa al núcleo intelectualista defendido por el estoicismo (con la posible excepción de Posidonio) a lo largo de

23 Cf. Philo Cher. 14-15 [LS 59H; SVF 3.513].

24 Si iuste depositum reddere in recte factis sit, in officiis ponatur depositum reddere; illo enim addito «iuste» fit recte factum, per se autem hoc ipsum reddere in officio ponitur. (Cic. Fin. 3.58-9 [LS 59F]).

25 Cf. Cic. Fin. 3.24-5 [LS 64H; SVF 3.11].

26 Stob. 2.93,14-18 [LS 59K; SVF 3.500].

27 Respecto de este último sentido en particular, $c f$. J. Sellars 2003.

$28 C f$. a modo de ejemplo, las interpretaciones recientes de D. Robertson 2010 y E. Buzaré 2011. 
toda su historia: desde una perspectiva monista, en efecto, la suposición de que ciertas estrategias no intelectuales (tales como la repetición, la memorización, etc.) son necesarias para alcanzar la virtud se vuelve sumamente peligrosa, en la medida en que tales estrategias parecen evidenciar necesariamente la existencia de procesos no reductibles al discurso racional. ${ }^{29}$ Las razones de que ello sea así son claras: una vez desterrada la existencia de partes irracionales del alma y habiendo traducido todo acontecimiento psíquico a un acontecimiento cognitivo, la única vía posible de modificación de la disposición del agente consiste en la demostración racional de la verdad de un determinando principio teórico. Como lo afirmará el discípulo más célebre del propio Musonio:

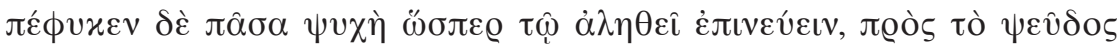

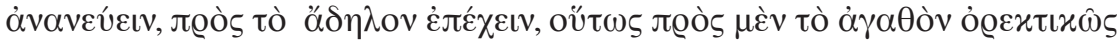

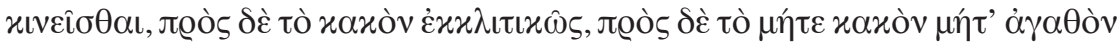

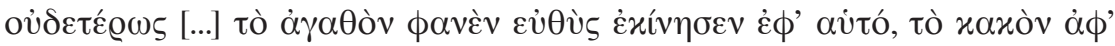

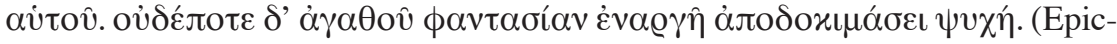
teto, Dissertationes 3.3.2-4). ${ }^{30}$

Toda alma, por naturaleza, igual que asiente a lo verdadero, niega lo falso y ante lo incierto se abstiene, así también ante el bien reacciona con deseo; ante el mal, con rechazo; ante lo que no es ni bueno ni malo, de ninguna de las dos maneras. [...] Cando se presenta el bien, inmediatamente se mueve hacia ello; cuando el mal, se aleja de ello. El alma nunca rechazará una impresión clara del bien. ${ }^{31}$

¿Descansan, entonces, D5 y D6, como parece temer Houser, ${ }^{32}$ sobre una concepción posidoniana de la habituación, ${ }^{33}$ una concepción que depende de la aceptación -contra la ortodoxia estoica-de la existencia de una parte irracional del alma (i.e., del sacrificio del monismo anímico en pos de una concepción de tipo platónico o aristotélico)? ¿En qué consiste, en última instancia, la ejercitación anímica que Musonio sugiere como un posible camino hacia la virtud?

29 Como se hace evidente, esta dificultad no afecta a Aristóteles, dado que el proceso de habituación en cuestión se refiere a las virtudes éticas, las cuales se vinculan explícitamente con la parte irracional del alma.

$30 C f$. asimismo Epicteto, Dissertationes 2.26.1-7.

31 Cito de acuerdo con W.A. Oldfather 1961; la traducción es de Ortíz Garcia (Epicteto 1993).

$32 C f$. A. Houser 1997:35-37.

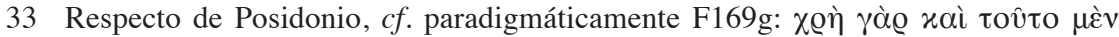

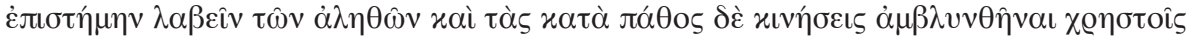

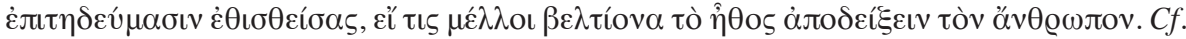
asimismo F31; F164; F165 (cito según la numeración de Kidd) y R. Sorabji 2000: passim. 
A juzgar por al menos dos pasajes similares que veremos a continuación, el proceso de habituación/ejercitación propuesto por Musonio parecería apoyarse en mecanismos no intelectuales. En efecto, si retomamos D6, la alusión a una continuidad entre el cuerpo y el alma en cuanto a los efectos que determinadas acciones pueden tener sobre ellos alude a un proceso donde la comprensión de principios teóricos no parece cumplir ningún papel:

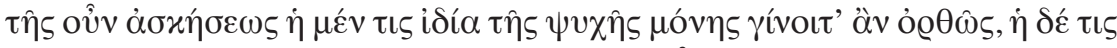

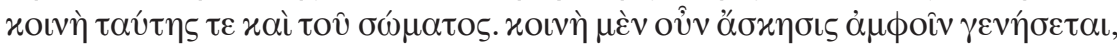

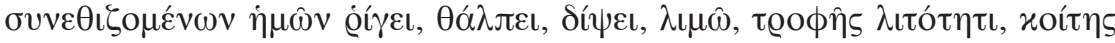

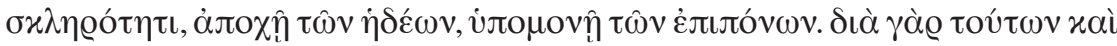

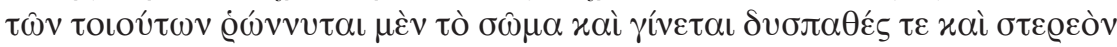

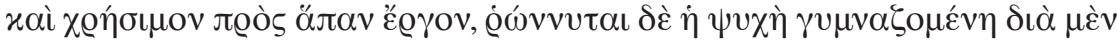

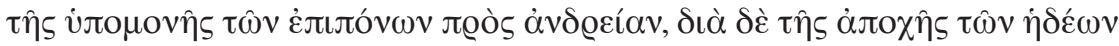

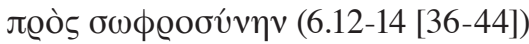

Un tipo de ejercitación es apropiado únicamente para el alma; otro tipo lo es para el cuerpo; otro es común para el alma y el cuerpo. Realizamos el ejercicio común a ambos [i.e., ejercitamos ambos] cuando nos habituamos a soportar el frío, el calor, el hambre, la sed, la escasez de comida, un lecho duro, a abstenernos de los placeres y a sobrellevar los sufrimientos. A través de estos métodos y otros parecidos, el cuerpo se fortalece, se vuelve capaz de tolerar los sufrimientos y apto para soportar cualquier tarea. El alma también se ve fortalecida, dado que se entrena en la valentía al sobrellevar los sufrimientos y se entrena en la moderación al abstenerse de los placeres. ${ }^{34}$

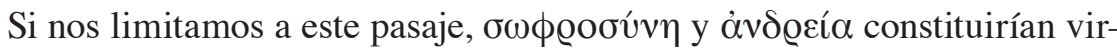
tudes cuya adquisición no requiere ningún proceso específico de comprensión intelectual, sino un simple proceso de habituación mecánica y repetitiva: tal como el estómago se termina habituando eventualmente y en forma automática a la escasez de alimentos, así el alma se vuelve moderada habituándose a abstenerse de los placeres. ${ }^{35} \mathrm{Si}$ sumamos a este pasaje unas líneas de la Disertación 4

34 Diógenes Laercio atribuye a Diógenes el Cínico una argumentación similar en DL 6.70-71. No obstante, aunque dicho pasaje coincide con D6 en distinguir, en primera instancia, entre los ejercicios corporales y los ejercicios anímicos para luego vincular a cierta clase de gimnasia corporal con la adquisición de la virtud, la similitud entre ambos pasajes es meramente superficial, en la medida en que el argumento de Diógenes consiste en señalar que para alcanzar

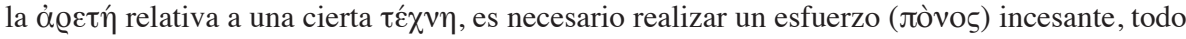
lo cual es, en el mejor de los casos, secundario para el argumento general de Musonio.

35 Hadot alude, analizando este pasaje, al hecho de que el gymnasion era el lugar en el que, además de los ejercicios físicos, se impartían con frecuencia las lecciones de filosofía ( $c f$. 
que refuerzan esta idea, el temor expresado por Houser respecto de que estemos ante una recaída platónico/posidoniana parece sumamente atendible:

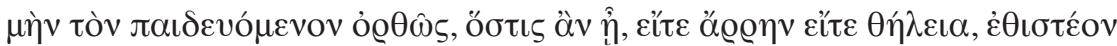

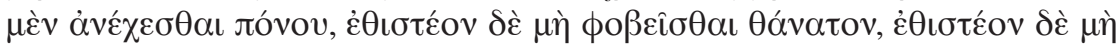

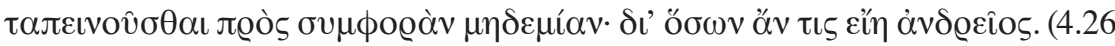
[78-82])

Un niño que es educado correctamente, ya sea varón o niña, debe ser habituado a tolerar las tribulaciones, a no temer a la muerte y a no ser derrotado por los infortunios; de esa forma será valiente.

\section{Los ejercicios intelectuales en la Disertación 6 y EPiCTeto}

El panorama que se ha ido construyendo hasta este punto (i.e., en la concatenación de D5 y D6.1-7) parece confirmar la idea compartida por buena parte de la crítica especializada respecto del carácter decisivo que asume la práctica en Musonio y respecto de la necesidad de concebir dicha práctica (o habituación o ejercitación) como un proceso que trasciende la mera comprensión teórica. No obstante, cuando llega el momento de ofrecer precisiones respecto de qué

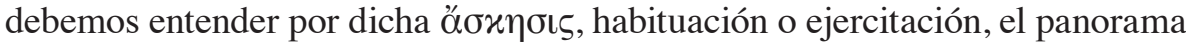
se modifica en forma decisiva:

6.8-9: [El proceso de ejercitación referido en 6.1-7] es más importante en la filosofía que en otras artes ( $\tau \dot{\varepsilon} \chi v \eta \varsigma)$ porque el hombre se inicia

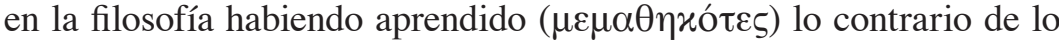
que debe ahora aprender y porque su alma está dañada a causa de la

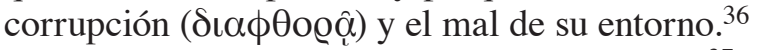

6.15: $\quad$ El ejercicio que es específico del alma ${ }^{37}$ consiste en mantener

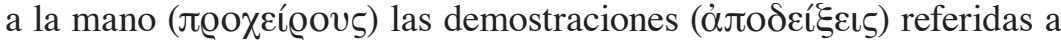
los bienes aparentes y en habituarse a reconocer ( $\gamma v \omega \varrho i ́ \zeta \varepsilon ı v) ~ l o s ~ b i e n e s$

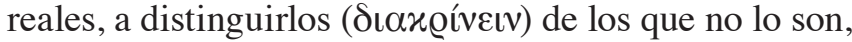

6.16: $\quad$ y en preocuparse ( $\mu \varepsilon \lambda \varepsilon \tau \alpha \hat{v})$ en no huir de los falsos males y en no perseguir los falsos bienes.

\footnotetext{
P. Hadot 1998:208).

36 Un análisis de esta problemática de la «corrupción» del ambiente en el que el individuo es educado se encuentra en R. Valantasis 1999.

37 Musonio distingue en D6.12-14 entre una ejercitación que es propia del alma, otra que es propia del cuerpo y otra que es común a los dos.
} 
Si tomamos en cuenta estas especificaciones, este ejercicio espiritual, como lo denomina Sellars, se vuelve un ejercicio esencialmente intelectual: la práctica en cuestión no es otra cosa, a fin de cuentas, que el acto de evaluar cada situación (problemática) a la luz del criterio ofrecido por la axiología estoica, esto es, la tripartición bueno/malo/indiferente, estando siempre atentos al hecho de que aquello que aparece prima facie como un bien o un mal puede no serlo. ${ }^{38} \mathrm{La}$ causa del vicio es, en el fondo, puramente intelectual: a pesar de que afirmamos estar de acuerdo con que nada que no sea el vicio debe ser temido y nada que no sea la virtud debe ser perseguido, «cuando sobrevienen los infortunios, pensamos ( $ү \gamma o u ́ \mu \varepsilon \theta \alpha$ ) que nos ha ocurrido algo malo; cuando viene el placer,

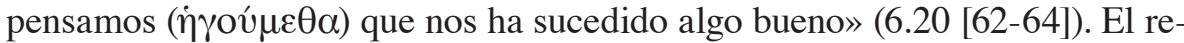
medio (i.e., el camino hacia la virtud) no puede ser, en consecuencia, otra cosa que una modificación de nuestros hábitos intelectuales: enfrentar cada situación armados con los principios apropiados para evaluarla correctamente (6.21 [69]:

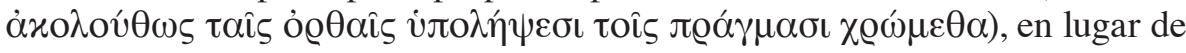
permitir que sean los principios heredados de nuestro entorno los que decidan sobre el valor de cada una de las alternativas que se abren ante nosotros.

Esto resuelve el interrogante acerca de si la insistencia de Musonio sobre los

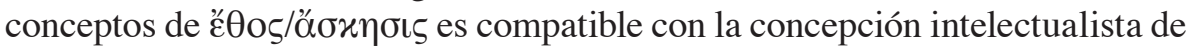
la acción humana defendida por el estoicismo, y disipa parcialmente los temores respecto de que D5-6 representen una recaída posidoniana. Queda sin resolver, a pesar de ello, una pregunta importante: ¿Por qué insiste Musonio en la idea de que debemos habituarnos a evaluar cada situación a través de los principios teóricos correctos, en lugar de establecer que debemos evaluar cada situación a través de dichos principios? ¿Qué es lo que aporta dicha habituación? En otras palabras: ¿en qué sentido la habituación a evaluar las situaciones armados con los principios teóricos apropiados puede producir que, eventualmente, alcancemos la virtud?

La respuesta a esta pregunta, desafortunadamente, no la podemos obtener directamente a partir de los escasos registros que se han conservado de las disertaciones de Musonio. No obstante, las Dissertationes de Epicteto pueden ser utilizadas, según propongo, para ensayar una respuesta provisoria. En ambas obras, en efecto, encontramos en forma recurrente una discusión análoga a la que hemos estado analizando en D5-6, presentada en términos virtualmente idénticos a los de Musonio ${ }^{39}$ y reiterando, en ciertos casos, estructuras argumentativas

38 Así como lo será en Epicteto la distinción entre lo que depende y lo que no depende de nostros, esta última advertencia representa el principio central sobre el que parecen haberse articulado las estrategias retórico-pedagógicas de Musonio.

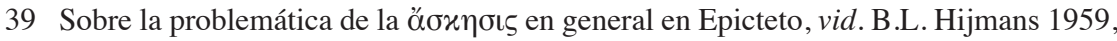
A.A. Long 2002 y, fundamentalmente, J. Cooper 2007. 
análogas. Tales paralelos, no obstante, coexisten con un aporte específico que

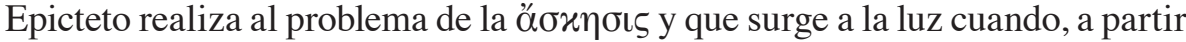
de la estructura aparentemente caótica de las lecciones recogidas por Arriano, reconstruimos una serie de estrategias sumamente coherentes y precisas que articulan en forma silenciosa la totalidad de las reflexiones de Epicteto referidas al progreso moral (i.e., al camino que recorre el proficiente en su trayecto hacia la virtud). Dichas estrategias se construyen fundamentalmente sobre dos ejes: en primer lugar, la distinción entre la mera comprensión abstracta de los principios teóricos y la aplicación de los mismos a los casos particulares; en segundo lugar, el énfasis por parte del autor en la necesidad de una comprensión analítica y sistemática de los conceptos que se hallan en juego en cada evaluación que realizamos de las situaciones a las que nos vemos enfrentados cotidianamente. ${ }^{40}$

Si incorporamos estos dos elementos, ${ }^{41}$ sugiero, podemos no sólo ofrecer una respuesta concreta al interrogante que señalábamos hace un momento como irresuelto en Musonio (i.e., en qué sentido la habituación a evaluar las situaciones que enfrentamos cotidianamente a la luz de los principios correctos puede contribuir a que eventualmente alcancemos la virtud), sino también contribuir a responder a otra pregunta, íntimamente vinculada a aquella, que todavía no hemos abordado, a saber: ¿en qué se distingue la acción del proficiente de la acción del individuo virtuoso?

La respuesta a ambos interrogantes puede ser presentada en forma unificada: a pesar de que una determinada acción realizada por un individuo virtuoso y uno que todavía no lo es pueden aparecer externamente como idénticas, la diferencia entre ambas acciones consiste en que la acción del individuo virtuoso, en primer lugar, no se funda sobre una comprensión puramente abstracta de ciertos principios teóricos, sino sobre la capacidad de aplicarlos correctamente

$40 C f$., a este respecto, Braicovich 2012 y 2013. Dissertationes 3.20 y 4.1.63-85 ofrecen dos ejemplos claros de este proceso complejo mediante el cual, partiendo de una idea de carácter general a la que el interlocutor asiente sin comprender todavía sus consecuencias lógicas, Epicteto despliega dialécticamente lo que significa adquirir una comprensión profunda de un principio teórico. Una aplicación sumamente interesante, desde el punto de vista pedagógico, de esta estrategia se encuentra en Dissertationes 2.19.20-34, donde Epicteto despliega las verdaderas consecuencias y el verdadero precio a pagar por ser un «filósofo estoico». $C f$. asimismo Dissertationes $3.16 .7-13$ y $1.22 .9 \mathrm{ss}$.

41 Esta estrategia tentativa de complementar las lagunas presentes en las disertaciones de Musonio con los desarrollos ofrecidos por Epicteto ciertamente supone que asumamos una continuidad doctrinal sustantiva entre ambos pensadores. Lo cierto, en este sentido, es que nada en las Dissertationes hace suponer que Epicteto se haya desviado en lo más mínimo respecto de las enseñanzas de su maestro, y las únicas divergencias evidentes son fundamentalmente temáticas, y son atribuibles a una diversidad de intereses entre ambos, y no a una diferencia en cuanto al enfoque doctrinal de cada uno. 
a la situación a la que se ve enfrentado; y, en segundo lugar, en que su acción se ve acompañada por una captación profunda y sistemática de las consecuencias lógicas que se derivan de dicha acción desde la perspectiva de la consistencia interna de su alma (i.e. de sus opiniones). La acción del individuo que todavía no ha alcanzado la virtud, por el contrario, carecerá de tales características (he ahí la respuesta a la segunda pregunta), pero la realización sostenida y sistemática del tipo correcto de acciones contribuirá a que el individuo adquiera gradualmente una comprensión cada vez más profunda de las múltiples dimensiones éticas que se derivan de sus acciones hasta que, eventualmente, las acciones que realice constituirán acciones correctas $(x \alpha \theta \eta \dot{\eta} \varkappa o v \tau \alpha)$ realizadas por los motivos correctos ( $x \alpha \tau o ́ \varrho \theta \omega \mu \alpha)$, o, puesto en términos aristotélicos, acciones realizadas a partir de una disposición virtuosa.

\section{CONCLUSIÓN}

Como señalé en la introducción, los objetivos específicos que me proponía en estas páginas eran los siguientes: en primer lugar, reconstruir en forma siste-

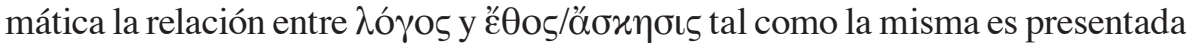
por Musonio en D5-6; en segundo lugar, postular las reflexiones de Aristóteles sobre el problema de la habituación como un marco conceptual relevante para encuadrar el análisis de D5-6; por último, analizar las posibles tensiones lógicas

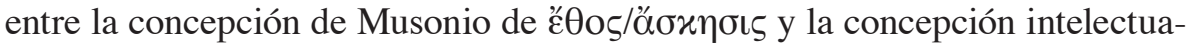
lista de la acción humana defendida por la ortodoxia estoica. Sugerí, de modo complementario, la posibilidad de recurrir a las Dissertationes de Epicteto en busca de desarrollos parciales que permitiesen ofrecer una respuesta tentativa a los interrogantes que no podemos responder a partir de la sola evidencia de D5-6. La conclusión fundamental que creo que es posible extraer a partir de este desarrollo consiste en el hecho de que la «práctica» frecuentemente señalada por los comentaristas como central para la concepción de la filosofía defendida por Musonio, puede legítimamente ser concebida como un proceso puramente intelectual, un proceso de captación cada vez más profunda y concreta de la teoría. Esto no agota, ciertamente, las tareas a realizar en la búsqueda de una comprensión más acabada de la concepción específica que los estoicos romanos desarrollaron de la actividad filosófica (de sus tareas, sus fundamentos y sus estrategias), pero permite al menos establecer una continuidad clara entre las reflexiones de Séneca, Musonio Rufo y Epicteto en cuanto al hecho de que en ninguno de los tres autores el énfasis en procesos activos y prácticos de reconstrucción de la subjetividad supone un recurso velado a una concepción dualista del alma ni un conflicto con la concepción intelectualista de la acción humana defendida por la ortodoxia estoica. 


\section{REFERENCIAS BIBLIOGRÁFICAS}

ARISTÓTELES. 2007: Ética nicomaquea, tr. Eduardo Sinnott. Buenos Aires: Ediciones Colihue.

BOWDITCH, N. 2008: «Aristotle on habituation: The key to unlocking the Nicomachean Ethics», Ethical Perspectives, 15.3, pp. 309-342.

BRAICOVICH, R.S. 2012: «Critical assent, intellectualism and repetition in Epictetus», Apeiron, 45, pp. 314-337.

, 2013: «Ejercicios espirituales e intelectualismo en Epicteto», Classica, 23.2.

[En prensa]

BROADIE, S. 1991: Ethics with Aristotle. Oxford: Oxford University Press.

BUZARÉ, E. 2011: Stoic Spiritual Exercises. North Carolina: Lulu.

BYWATER, I. 1962: Aristotelis. Ethica Nicomachea. Oxford: Clarendon Press.

COOPER, J.M. 2007: «The Relevance of Moral Theory to Moral Improvement in Epictetus», en A.S. Mason y Th. Scaltsas (eds.), The Philosophy of Epictetus. Oxford: Oxford University Press, pp. 9-19.

EPICTETO. 1993: Disertaciones, tr. Paloma Ortiz García. Barcelona: Gredos.

FOUCAULT, M. 1994: Histoire de la sexualité, tome 3: Le souci de soi. París: Gallimard.

GILL, C. 2006: The Structured Self in Hellenistic and Roman Thought. Oxford: Oxford University Press.

GOTTLIEB, P. 2009: The Virtue of Aristotle's Ethics. Cambridge: Cambridge University Press.

HADOT, P. 1993: Exercices spirituels et philosophie antique. Paris: Albin Michel. , 1998: ¿Qué es la filosofía antigua?. México: Fondo de Cultura Económica.

HIJMANS, B.L. 1959: Askesis. Notes on Epictetus' Educational System. Assen.

HOUSER, J.S. 1997: The Philosophy of Musonius Rufus. A Study of Applied Ethics in Late Stoa. Tesis Doctoral. Brown University.

HURSTHOUSE, R. 1988: «Moral Habituation. A review of Troels Engberg-Pedersen, Aristotle's Theory of Moral Insight», Oxford Studies in Ancient Philosophy, 6, pp. 201-19.

INWOOD, B. 1985: Ethics and Action in Early Stoicism. Oxford: Clarendon Press. , 2004: Reseña de The Art of Living: the Stoics on the Nature and Function of Philosophy, de John Sellars. Notre Dame Philosophical Reviews, 04 de Abril, http://ndpr.nd.edu/news/23760-the-art-of-living-the-stoics-on-the-nature-andfunction-of-philosophy.

KING, C. 2011: Musonius Rufus: Lectures and Sayings. CreateSpace.

LONG, A.A. 2002: Epictetus. A Stoic and Socratic Guide to Life. Oxford: Oxford University Press.

LUTZ, C. 1947: «Musonius Rufus: The Roman Socrates», Yale Classical Studies, 10, pp. 3-147.

MASON, A.S.; Scaltsas, T. (eds.). 2007: The Philosophy of Epictetus. Oxford: Oxford University Press. 
MORFORD, M.. 2002: The Roman Philosophers. From the Time of Cato the Censor to the Death of Marcus Aurelius. Londres: Routledge.

NUSSBAUM, M. 1996: The Therapy of Desire. Theory and Praxis in Hellenistic Ethics. Princeton: Princeton University Press.

OLDFATHER, W.A. 1961: Epictetus: The Discourses as reported by Arrian. The Manual and Fragments. Londres: Heinemann.

POMEROY PARKER, Ch. 1896: «Musonius the Etruscan», Harvard Studies in Classical Philology, 7, pp. 123-137.

REYDAMS-SCHILS, G. 2005: The Roman Stoics. Self, Responsibility, and Affection. Chicago: University of Chicago Press.

ROBERTSON, D. 2010: The philosophy of cognitive-behavioural therapy (CBT) stoic philosophy as rational and cognitive psychotherapy. Londres: Karnac Books.

ROSKAM, G. 2005: On the Path to Virtue. The Stoic Doctrine of Moral Progress and its Reception in (Middle-)Platonism. Leuven: Leuven University Press.

SELLARS, J. 2003: The Art of Living: the Stoics on the Nature and Function of Philosophy. Ashgate.

, 2007: «Stoic practical philosophy in the imperial period», Greek and Roman Philosophy, 100 BC-200 AD, Bulletin of the Institute of Classical Studies, Supl. 94.1, pp. 115-140

SORABJI, R. 1973: «Aristotle on the role of intellect in virtue», Proceedings of the Aristotelian Society, 74, pp. 107-129.

SORABJI, R. 2000: Emotion and Peace Of Mind. From Stoic Agitation to Christian Temptation. The Gifford Lectures. Cambridge: Cambridge University Press.

VALANTASIS, R. 1999: «Musonius Rufus and Greco-Roman ascetical theory», Greek, Roman and Byzantine Studies, 40.3, pp. 207-231.

Rodrigo SEBASTIÁN BRAICovich pertenece al Consejo Nacional de Investigaciones Científicas y Técnicas (CONICET), Argentina

Líneas de investigación:

La concepción intelectualista de la acción humana en el estoicismo romano y su vinculación con las estrategias terapéuticas desarrolladas por Séneca, Musonio Rufo, Epicteto y Marco Aurelio.

Publicaciones recientes:

(2010) "Freedom and epistemic determinism in Epictetus' Discourses", The Classical Quarterly (Oxford), 60.1, pp. 202-220. ISSN: 0009-8388.

(2012) "Critical assent, intellectualism and repetition in Epictetus", Apeiron: Journalfor Ancient Philosophy and Science (De Gruyter). [Aceptado para publicación]. ISSN: 0003-6390.

Dirección electrónica: rbraicovich@gmail.com 